\title{
Back-to-Back Test of Reconfigurable Add- Drop Using a Silicon Photonics Microchip (1525090-Y2)
}

\author{
SHAYAN MOOKHERJEA \\ Department of Electrical and Computer Engineering, University of California, San Diego, MC 0407 La \\ Jolla CA 92093-0407 USA \\ Email:smookher@ucsd.edu
}

Summary of a Project Outcomes report of research funded by the U.S. National Science Foundation under Project Number 1525090 (Year 2). We study the design of compact headend components at the transceiver level using silicon photonics to implement disaggregation for improving optical communications, and demonstrate novel functionality at the link level.

\section{Introduction and Goals}

A disaggregated network collocates some of the various computing, memory, storage, and networking resources for higher efficiency, and its benefits include enabling statistical multiplexing, greater operator control and enabling software-defined networking [1], [2]. Disaggregation is a broad and general concept; in this project, the key envisioned demonstration that will be enabled by disaggregation is adaptability of physical-layer resources to the traffic demand. Bandwidth is a key resource of optical communication links and a major advantage over electronic links which typically do not use bandwidth multiplexing as widely.

An introduction to this NSF-funded project and its objectives was provided in an earlier report. To summarize, the principal goal of this project is to study and develop a practical method for implementing disaggregation and the role of optics hardware. Specifically, we proposed a way to implement disaggregation in a practical sense (without additional fibers or input/output ports) by designing a small, head-end component at the transceiver level using silicon photonics.

In this project, disaggregation is implemented through control over the spectrum: Control bits will be inserted at and extracted from a small, controlled wavelength shift from the data channel. We design and operate a silicon photonic microchip in which the control and monitoring signals are delivered from a remote controller using optical wavelengths (lambdas) at different parts of the spectrum than where the data channels are located which would allow control/routing/grooming signals to transported and distributed optically rather than electronically.

This overcomes the latency-utilization-cost tradeoffs for conventional communication architectures, and is of current interest for network designers and computer communication system architects. For example [3], "flexible-grid reconfigurable optical add-drop multiplexers, bandwidth-variable transceivers, 
and the ability to choose a variety of optical source types are integral for cloud network operators to improve network efficiency while supporting a variety of service types."

\section{Strategy}

In this project, disaggregation is implemented through the spectral degree of freedom, underutilized in data communication networks and coarse WDM systems. We propose to use optics to carry control and monitoring signals between hosts and the controller unit, wherever it may be located on the network. Optical fiber is a suitable technology for decoupling the inherent distance-energy-latency tradeoffs of electronic wires.

Also, we compensate for the potentially increased number of logical interfaces without increasing the number of fibers. This can be managed if we copropagate the new signals over low-bandwidth, spectrally-separated "lambdas" on the same fibers. While not being the only possible solution, this is quite elegant in silicon photonics. It leverages the well-known strengths of this platform in terms of devices with high bandwidth and small footprint, which can be formed into complex integrated photonic structures that control the flow of light.

\section{Activities and Impact}

Last year, we showed how to extract and insert discrete laser wavelengths from a common "bus" fiber. Our prototypical filter architecture is based on single microring resonators [4], [5] or one that is based on the coupled-resonator optical waveguide concept [6]. There is interesting physics in how light propagates in such a structure [7] (based on the coupling of optical microresonators [8]) which affects the dispersion characteristics of the channels [9], [10]. One of the potential drawbacks of microresonators is that their performance in the nonlinear regime is different than in the linear regime [11][14].

One of the research tasks performed this year was the demonstration of the ability to drop and add modulated data rather than continuous-wave light from a laser. Figure 1 shows the results of a test performed on a campus-level network, in which a spectral band was "dropped" (i.e., filtered out), with a reduction in optical power by at least a factor of 10 . The dispersion of the coupler between the filter and the bus waveguide is especially important to achieve wideband performance [15]-[17]. The grey and blue lines show the spectrum before and after the chip, with post-chip amplification performed using an EDFA to compensate for the fiber-to-waveguide insertion loss. The test chip was not packaged, and relied on a micropositioner-driven alignment 
of an optical fiber array to the diced chip edge for optical input / output, which incurred higher losses than in a packaged chip with optimized couplers.

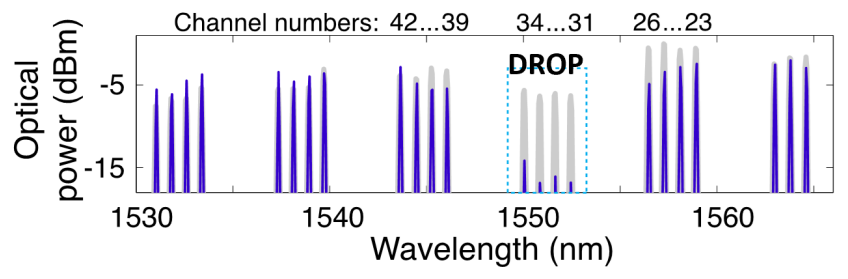

Neighboring channels:
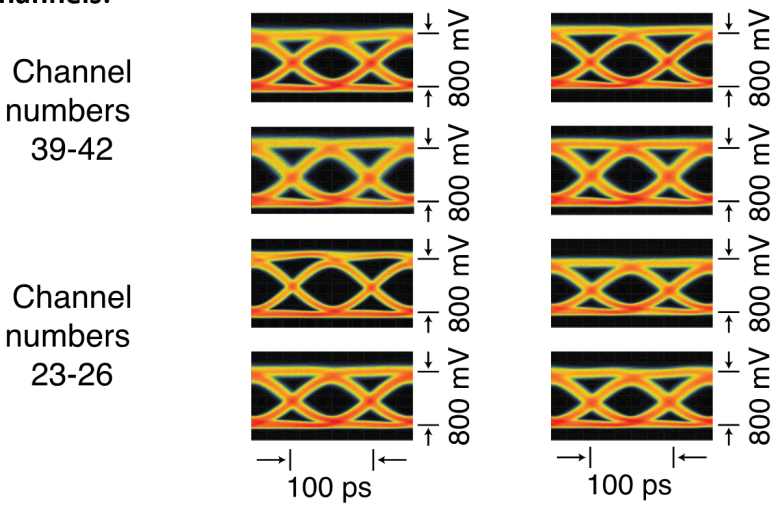

Figure 1 A group of four channels out of twenty channels (each carrying $10 \mathrm{Gbit} / \mathrm{s}$ NRZ data generated by a computer server equipped with an SFP+ transceiver) in a ring network was dropped using the silicon photonic chip chip, to clear out a portion of the spectrum for subsequent 'add'. The eye diagrams of the neighboring channels are shown to be undisturbed.

Figure 2 shows the results of adding a modulated data channel using the same silicon photonic circuit. The microchip is capable of adding four such channels. Because of the imperfect filtering in this version of the chip (as seen by the extinction ratio limited to about $10 \mathrm{~dB}$ in Fig. 1), we observed some penalty compared to back-to-back transmission, but this can be improved in future fabrication iterations with improved filter design. Emerging devices with a greater spectral utilization are likely to place a higher burden on the filters [18].

This year, we also showed how to vary the bandwidth of the spectral slices, which is an example of permitting the adaptability of physical resources on demand in order to allow software-defined or statistical traffic multiplexing. Overprovisioned optical links are wasteful and present a photonic equivalent of today's "dark silicon" problem whereby a significant fraction of the hardware resources must remain under-utilized because of energy cost constraints.

Silicon microresonators are sensitive to fabrication imperfections and we have studied how to fabricate, test and improve devices [19] perhaps using infrared imaging techniques and post-fabrication compensation methods [20]-[22].

The optical loss of the microchip (fiber to fiber) is about $10 \mathrm{~dB}$ and external optical amplification was used to compensate for this loss and provide 
sufficient power for the eye diagram and bit error rate measurements. If used, the CROW architecture can provide gain using current injection which may be useful for compensating the optical insertion loss [23], [24].
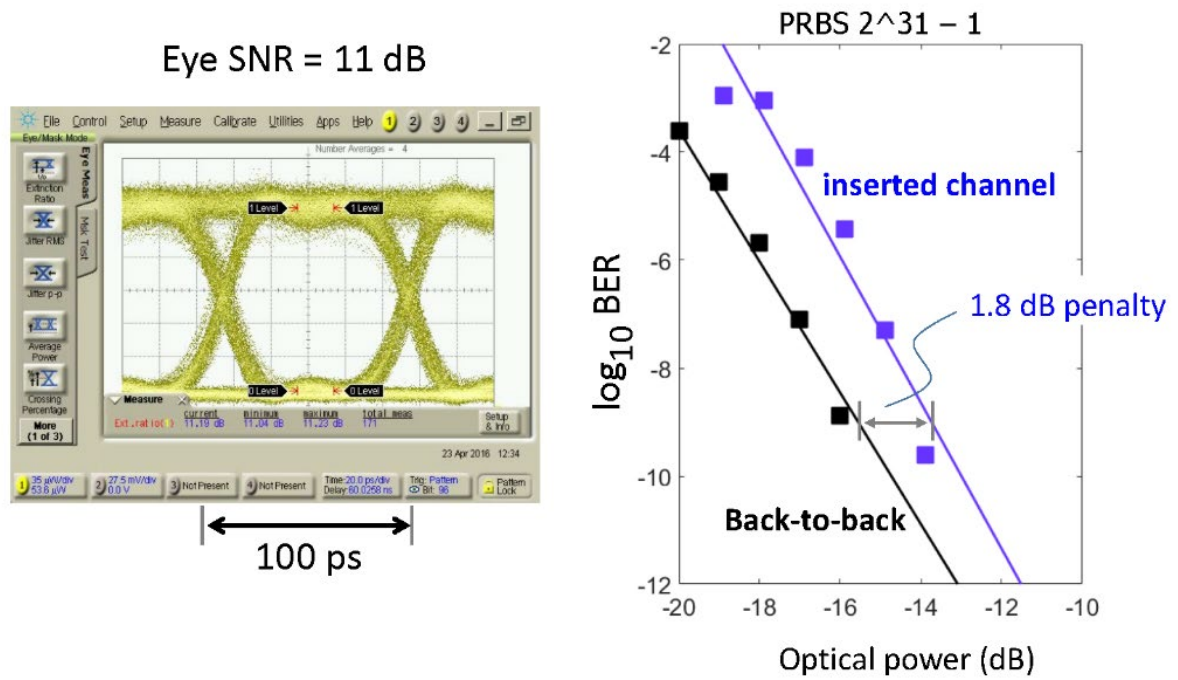

Figure 2 Clean eye diagram and bit-error-rate test at $10 \mathrm{Gbit} / \mathrm{s}$ NRS (2^31-1) PRBS showing less than $2 \mathrm{~dB}$ penalty compared to no-chip (back-to-back) transmission incurred by adding a channel.

We also study whether we can use silicon photoncis to implement quantum side communication channels over the same optical fiber. Similar devices have been used for wavelength conversion (using four-wave mixing) integrated with filtering [25], and for generating entangled photon pairs (using spontaneous four-wave mixing) [26]-[28]. The intersection of dispersive propagation and nonlinear optics is especially rich in physics [29], [30], and can lead to insights on controlling light propagation in tightly-confined microstructures.

Last year, we published two submitted journal publications (Optics Express) from our first round of measurements on test chips. This year, results were presented at the CLEO conference, and also at an OSA/OIDA workshop held at OFC.

\section{Open-Access Reporting Initiative}

PRAISE: This open-access document is provided in support of our PRAISE

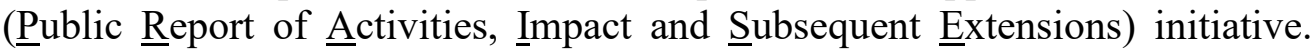
What is it? An open-access document shared with the public which describes the research outcomes of publicly-funded projects such as those funded by the U.S. NSF (National Science Foundation).

\section{References}


[1] S. Han, N. Egi, A. Panda, S. Ratnasamy, G. Shi, and S. Shenker, "Network support for resource disaggregation in next-generation datacenters," in Proceedings of the Twelfth ACM Workshop on Hot Topics in Networks, College Park Maryland, Nov. 2013, pp. 1-7. doi: 10.1145/2535771.2535778.

[2] J. Lee et al., "Application-driven bandwidth guarantees in datacenters," in Proceedings of the 2014 ACM conference on SIGCOMM, Chicago Illinois USA, Aug. 2014, pp. 467-478. doi: 10.1145/2619239.2626326.

[3] M. Filer et al., "Elastic Optical Networking in the Microsoft Cloud [Invited]," J. Opt. Commun. Netw., vol. 8, no. 7, p. A45, Jul. 2016, doi: 10.1364/JOCN.8.000A45.

[4] R. Aguinaldo et al., "Wideband silicon-photonic thermo-optic switch in a wavelength-division multiplexed ring network," Opt. Express, vol. 22, no. 7, p. 8205, Apr. 2014, doi: 10.1364/OE.22.008205.

[5] R. Aguinaldo, Yiran Shen, and S. Mookherjea, "Large Dispersion of Silicon Directional Couplers Obtained via Wideband Microring Parametric Characterization," IEEE Photon. Technol. Lett., vol. 24, no. 14, pp. 1242-1244, Jul. 2012, doi: 10.1109/LPT.2012.2198639.

[6] M. L. Cooper et al., "235-ring Coupled-Resonator Optical Waveguides," in Conference on Lasers and Electro-Optics 2010, San Jose, California, 2010, p. CTuHH3. doi: 10.1364/CLEO.2010.CTuHH3.

[7] S. Mookherjea and A. Yariv, "Optical pulse propagation in the tightbinding approximation," Opt. Express, vol. 9, no. 2, p. 91, Jul. 2001, doi: 10.1364/OE.9.000091.

[8] S. Mookherjea, "Spectral characteristics of coupled resonators," J. Opt. Soc. Am. B, vol. 23, no. 6, p. 1137, Jun. 2006, doi: 10.1364/JOSAB.23.001137.

[9] S. Mookherjea and A. Yariv, "Pulse propagation in a coupled resonator optical waveguide to all orders of dispersion," Phys. Rev. E, vol. 65, no. 5, p. 056601, Apr. 2002, doi: 10.1103/PhysRevE.65.056601.

[10] S. Mookherjea, D. S. Cohen, and A. Yariv, "Nonlinear dispersion in a coupled-resonator optical waveguide," Opt. Lett., vol. 27, no. 11, p. 933, Jun. 2002, doi: 10.1364/OL.27.000933.

[11] S. Mookherjea and M. A. Schneider, "The nonlinear microring adddrop filter," Opt. Express, vol. 16, no. 19, p. 15130, Sep. 2008, doi: 10.1364/OE.16.015130.

[12] S. Mookherjea and A. Yariv, "Kerr-stabilized super-resonant modes in coupled-resonator optical waveguides," Phys. Rev. E, vol. 66, no. 4, p. 046610, Oct. 2002, doi: 10.1103/PhysRevE.66.046610.

[13] J. R. Ong et al., "Low-power continuous-wave four-wave mixing in silicon coupled-resonator optical waveguides," Opt. Lett., vol. 36, no. 15, pp. 2964-2966, 2011. 
[14] S. Mookherjea and A. Yariv, "Optical pulse propagation and holographic storage in a coupled-resonator optical waveguide," Phys. Rev. $E$, vol. 64, no. 6, p. 066602 , Nov. 2001, doi: 10.1103/PhysRevE.64.066602.

[15] M. L. Cooper and S. Mookherjea, "Numerically-assisted coupledmode theory for silicon waveguide couplers and arrayed waveguides," Opt. Express, vol. 17, no. 3, p. 1583, Feb. 2009, doi: 10.1364/OE.17.001583.

[16] S. Mookherjea and M. A. Schneider, "Avoiding bandwidth collapse in long chains of coupled optical microresonators," Opt. Lett., vol. 36, no. 23, p. 4557, Dec. 2011, doi: 10.1364/OL.36.004557.

[17] M. L. Cooper and S. Mookherjea, "Modeling of Multiband Transmission in Long Silicon Coupled-Resonator Optical Waveguides," IEEE Photon. Technol. Lett., vol. 23, no. 13, pp. 872-874, Jul. 2011, doi: 10.1109/LPT.2011.2141657.

[18] X. Wang, P. O. Weigel, J. Zhao, M. Ruesing, and S. Mookherjea, "Achieving beyond-100-GHz large-signal modulation bandwidth in hybrid silicon photonics Mach Zehnder modulators using thin film lithium niobate," APL Photonics, vol. 4, no. 9, p. 096101, Sep. 2019, doi: $10.1063 / 1.5115243$.

[19] S. Mookherjea, J. R. Ong, X. Luo, and L. Guo-Qiang, "Electronic control of optical Anderson localization modes," Nature Nanotech, vol. 9, no. 5, pp. 365-371, May 2014, doi: 10.1038/nnano.2014.53.

[20] M. L. Cooper, G. Gupta, J. S. Park, M. A. Schneider, I. B. Divliansky, and S. Mookherjea, "Quantitative infrared imaging of silicon-on-insulator microring resonators," Opt. Lett., vol. 35, no. 5, p. 784, Mar. 2010, doi: 10.1364/OL.35.000784.

[21] Y. Shen, I. B. Divliansky, D. N. Basov, and S. Mookherjea, "Perfect set-and-forget alignment of silicon photonic resonators and interferometers," in Optical Fiber Communication Conference/National Fiber Optic Engineers Conference 2011, Los Angeles, California, 2011, p. PDPC3. doi: 10.1364/OFC.2011.PDPC3.

[22] S. Mookherjea and H. R. Grant, "High dynamic range microscope infrared imaging of silicon nanophotonic devices," Opt. Lett., vol. 37, no. 22, p. 4705, Nov. 2012, doi: 10.1364/OL.37.004705.

[23] S. Mookherjea, "Semiconductor coupled-resonator optical waveguide laser," Appl. Phys. Lett., vol. 84, no. 17, pp. 3265-3267, Apr. 2004, doi: 10.1063/1.1719278.

[24] S. Mookherjea, "Using gain to tune the dispersion relation of coupledresonator optical waveguides," IEEE Photon. Technol. Lett., vol. 18, no. 5, pp. 715-717, Mar. 2006, doi: 10.1109/LPT.2006.871144. 
[25] J. R. Ong, R. Kumar, and S. Mookherjea, "Silicon microring-based wavelength converter with integrated pump and signal suppression," Opt. Lett., vol. 39, no. 15, p. 4439, Aug. 2014, doi: 10.1364/OL.39.004439.

[26] J. R. Ong and S. Mookherjea, "Quantum light generation on a silicon chip using waveguides and resonators," Opt. Express, vol. 21, no. 4, p. 5171, Feb. 2013, doi: 10.1364/OE.21.005171.

[27] M. Savanier, R. Kumar, and S. Mookherjea, "Optimizing photon-pair generation electronically using a $p-i-n$ diode incorporated in a silicon microring resonator," Appl. Phys. Lett., vol. 107, no. 13, p. 131101, Sep. 2015, doi: 10.1063/1.4932047.

[28] R. Kumar, M. Savanier, J. R. Ong, and S. Mookherjea, "Entanglement measurement of a coupled silicon microring photon pair source," Opt. Express, vol. 23, no. 15, p. 19318, Jul. 2015, doi: 10.1364/OE.23.019318.

[29] A. Ciattoni, B. Crosignani, S. Mookherjea, and A. Yariv, "Nonparaxial dark solitons in optical Kerr media," Opt. Lett., vol. 30, no. 5, p. 516, Mar. 2005, doi: 10.1364/OL.30.000516.

[30] B. Crosignani, A. Yariv, and S. Mookherjea, "Nonparaxial spatial solitons and propagation-invariant pattern solutions in optical Kerr media," Opt. Lett., vol. 29, no. 11, p. 1254, Jun. 2004, doi:

10.1364/OL.29.001254. 